\title{
GESTIÓN INTEGRADA DEL RECURSO HÍDRICO EN LA CUENCA MAURE- UCHUSUMA-CAPLINA DE LA REGIÓN TACNA; Y EL DESARROLLO DE UNA MINERÍA SOSTENIBLE EN LA ZONA
}

Responsable: MSc. Dante Manzanares Cáceres Miembro: Ing. José Rodriguez Copare

\section{RESUMEN}

El proyecto de investigación propuesto sólo ha alcanzado el nivel de diagnóstico. En esta fase inicial ha consistido en la recopilación de la información sobre las cuencas. Consideramos la pertinencia del proyecto propuesto debido a que en todos los estudios elaborados hasta la fecha, respecto a la gestión de los recursos hídricos de estas cuencas, no han tomado en consideración la demanda real ni potencial del sector minero, y se desaprovecha la oportunidad de incorporar nuevas fuentes y caudales al inventario de dichos recursos.

Respecto a la propuesta básica de una Minería Sostenible en la Zona, se requiere: Determinar la demanda potencial de recursos hídricos por el sector minero, en función de la cantidad y calidad de las reservas de minerales determinadas en la etapa de exploración. Considerando, además, la fortaleza y oportunidad que ofrece el descubrimiento de reservas auríferas, que para su recuperación demandan limitadas cantidades de recurso hídrico, constituir Mesas de Trabajo intersectoriales que permitan negociar el aporte de las empresas mineras para mejorar la oferta de los recursos hidricos del subsuelo, en el flanco occidental de la Cordillera del Barroso, y el tratamiento de las aguas subsuperficiales contaminadas de manera natural, para que alcancen calidad de uso.
ABSTRACT

The proposed Project of Investigation has only reached the level of Diagnosis. The research in the initial phase has consisted of the compilation of the information on the river basins. We considered the pertinence of the Proposed Project, due to which in all the studies elaborated to the date with respect to the management of the hydric resources of these river basins they have not taken in consideration the real nor potential demand of the mining sector and the opportunity is failed to take advantage of to incorporate new sources and volumes to the inventory of these resources.

With respect to the basic proposal of a Sustainable Mining in the Zone, it is required: To determine the potential demand of hydric resources by the mining sector based on the amount and quality of the mineral reserves determined in the exploration stage. Considering in addition, the strength and opportunity which it offers the discovery of auriferous reserves, that for its recovery they demand limited amounts of hydric resource, and to constitute work groups that allow to negotiate the contribution of the mining companies to improve the supply of the hydric resources of the subsoil, in the western flank of the Mountain range of the Muddy one, and the treatment of sub superficial waters contaminated of natural way, so that they reach quality of use.

\section{INTRODUCCIÓN}

El agua es un recurso imprescindible para el desarrollo de los pueblos. Es un recurso unitario por lo que los aspectos de cantidad, calidad y oportunidad deben ser vistos de manera integral. Asimismo, se trata de un recurso fácilmente vulnerable por las actividades humanas. Los organismos mundiales han advertido que si la humanidad no realiza cambios importantes en sus usos y costumbres, en el año 2025 existirán muchas zonas de Latinoamérica (Perú, Chile), Africa y Asia, entre otros en los que se desencadenarán agudos conflictos por la escasez del agua. Por ello se están concertando políticas globales para revertir este proceso y poder asegurar el futuro de las nuevas generaciones, que tienen derecho a recibir un mundo que les dé iguales o mejores oportunidades que las que nosotros tuvimos.

La gestión de los recursos hídricos en nuestro pais, y en especial en nuestra Región Tacna, se desarrolla actualmente en torno al sector agrario, surgiendo la imperiosa necesidad de alentar un enfoque integral orientado a la coordinación de las intervenciones para el aprovechamiento multisectorial del agua, considerándolo como un bien económico, cuyo manejo debe basarse en los criterios de eficiencia, equidad y sostenibilidad. Lo expuesto resulta especialmente crítico en nuestra Región Tacna, por cuanto el recurso hídrico se constituye en el aspecto ambiental más significativo por el desbalance hidrico existente entre la disponibilidad y el requerimiento del mismo. lo 
cual se está agravando cada vez mas por encontrarnos en la cabecera del desierto de Atacama y la notoria regresión de nuestras nieves perpetuas en la zona alto andina de nuestra Región como consecuencia del cambio climático global.

\section{HIPOTESIS}

Es posible una gestión integrada del recurso hídrico en la cuenca Maure.UchusumaCaplina, mediante el desarrollo de sistemas de gestión técnica para la asignación óptima de los recursos hídricos a nivel de la cuenca, innovando herramientas de gestión ambiental, social y económica del agua, compatible con el desarrollo de una Minería sostenible en la zona.

\section{OBJETIVOS DE LA INVESTIGACIÓN:}

Determinar la estructura y funcionamiento de un modelo que permita el suministro de agua garantizando el acceso de todos los usuarios, satisfaciendo sus necesidades en cantidad, calidad y oportunidad ello promoviendo la concertación y la participación de todos los actores, la preservación y conservación del medio ambiente y el desarrollo social enmarcado en el concepto de desarrollo humano sostenible.

Identificación e innovación de herramientas de gestión ambiental, social y económica del agua en la cuenca en estudio, así como la adaptación y desarrollo de tecnologías para la gestión y uso eficiente del agua para consumo poblacional, en agricultura, minería y otras actividades en la cuenca.

\section{JUSTIFICACIÓN DEL PROBLEMA:}

Los resultados de la investigación constituirán una contribución importante desde la ciencia y la tecnología para reajustar las politicas y acciones que se aplican hasta la fecha sobre el aspecto ambiental más importante de la Región Tacna. En especial en la cuenca que abastece a la mayor población y sectores agrarios e industriales de la Región.

\section{METODOLOGÍA}

La investigación se caracteriza como bibliográfica en su primera fase. El área de estudio está constituida por las cuencas de los ríos Maure, Uchusuma y Caplina. En la segunda fase, se aplicarán métodos y técnicas cualitativas.

Discusión sobre el Diagnóstico de las cuencas Maure-Uchusuma y Caplina: Los Resultados obtenidos en el Diagnóstico de las Cuencas Maure-Uchusuma y Caplina permiten establecer un conjunto de tareas conducentes al manejo integrado de las mismas, estableciéndose un conjunto de indicadores que permitan controlar el desempeño de las mismas.

Dichas tareas forman parte del Plan de Manejo de las cuencas Maure-Uchusuma y Caplina, que sería implementada por los sectores integrados en la Comisión Ambiental Regional, abarcando los siguientes elementos:

\section{a. Recursos Hidricos Disponibles [Actual y Potencial]}

\begin{tabular}{|c|c|c|c|c|c|}
\hline \multirow{2}{*}{\multicolumn{2}{|c|}{ DESCARGAS }} & \multicolumn{4}{|c|}{ DESCARGAS DEL RIO CAPLINA Y UCHUSUNA BAJO } \\
\hline & & \multicolumn{2}{|c|}{ Rio Caplina } & \multicolumn{2}{|c|}{ Uchusuma Bajo } \\
\hline Matxima Controlada & $m^{1 / s}$ & 33,21 & febrero & 11,06 & marzo \\
\hline Descarga Minima & $\mathrm{m}^{2} / \mathrm{s}$ & 0,115 & $\operatorname{marzo}$ & 0,00 & septiembre \\
\hline Media Arual Promedio & $\mathrm{m}^{1 / 3}$ & 0,935 & & 0,586 & \\
\hline Masa Total Anual & MMC & \multicolumn{2}{|c|}{29,19} & \multicolumn{2}{|c|}{18,45} \\
\hline Promedio Mensuales (75\%) & $m^{1 / s}$ & \multicolumn{2}{|c|}{0,602} & \multicolumn{2}{|c|}{0,389} \\
\hline Volumen Total Anual (75\%) & MMC & \multicolumn{2}{|c|}{18,94} & \multicolumn{2}{|c|}{12,26} \\
\hline \multicolumn{2}{|c|}{ Meses Concentración Descargas } & \multicolumn{2}{|c|}{ Enero-Marzo } & \multicolumn{2}{|c|}{ Febrero-Marzo } \\
\hline \multicolumn{2}{|l|}{ Mese Periodo de Escasez } & \multicolumn{2}{|c|}{ Agosto - Noviembre } & \multicolumn{2}{|c|}{ Octubre - Noviembre } \\
\hline $\begin{array}{l}\text { Rendimiento Medio Anual } \\
\text { Cuenca Hümeda }\end{array}$ & its por km ' & \multicolumn{2}{|c|}{1,71} & \multicolumn{2}{|c|}{ s/d } \\
\hline
\end{tabular}

\section{b. Balance Hídrico Recursos Hídricos [MMC Anuales]}

\begin{tabular}{|l|c|c|c|c|}
\hline \multicolumn{1}{|c|}{ Usuario } & $\begin{array}{c}\text { Caplina - } \\
\text { Uchusuma }\end{array}$ & Sama & Locumba & Total \\
\hline Poblacional & 23,0 & 1,0 & 2,0 & 26,0 \\
\hline Riego & 45,0 & 47,0 & 43,0 & 135,0 \\
\hline Otros & 0,3 & $\cdot$ & 24,0 & 24,3 \\
\hline Derrianda total & 68,3 & 48,0 & 69,0 & 185,3 \\
\hline $\begin{array}{l}\text { Oferta } \\
\text { Superficial }\end{array}$ & 31,0 & 21,0 & 59,0 & 111,0 \\
\hline $\begin{array}{l}\text { Oferta } \\
\text { Subterránea }\end{array}$ & $57 \cdot 73$ & N.D. & N.D. & \\
\hline Déficit & $27 \%$ & $30 \%$ & $13 \%$ & $22,6 \%$ \\
\hline
\end{tabular}

\section{Disponibilidad de agua:}

Red de monitoreo meteorológico e hidrológico: lagunas, ríos, represas y aguas subterráneas.

Seguimiento al monitoreo de la red de control hidrogeológico: cantidad y calidad y nivel.

Construcción de infraestructura para almacenamiento de agua: disponibilidad hidrica y posibilidad de embalses (Caplina y Uchusuma).

Mecanismo de regulación de caudales e implementación de vasos reguladores.

\section{Sostenibilidad de los pozos del Ayro.}

Uso sostenible del acuifero de La Yarada:

- Estudio geofísico de refracción:

- Estudio hidrogeológico de La Yarada: lo tiene el PET y el INRENA (ATDR) 
- Diagnóstico de la demanda hídrica: ATDR lo entrega.Mejora en la eficiencia del uso del agua.

- Cobertura vegetal cuenca alta, para lacaptación de agua.

- Elaboración de diagnóstico de la oferta de agua:Diagnóstico de fuentes de agua: (inventario actualizado-PET).

\section{Eficiencia de inversiones:}

Diseño adecuado de proyectos.

Seguimiento a la ejecución de proyectos.

\section{Uso del agua:}

Diagnóstico de la demanda multisectorial del agua.

- Diagnóstico de la demanda de agua para uso minero y destino de efluentes.

- Diagnóstico de la demanda de agua para uso industrial y destino de efluentes.

- Diagnóstico de la demanda de agua para uso poblacional y destino de efluentes

- Diagnóstico de la demanda de agua para uso agrícola y destino de efluentes.

Reducción del uso informal del agua.

- Reducción de predios agrícolas que usan agua sin estar en el padrón de uso agrícola.

- Reducción de pozos informales.

- Desarrollar un sistema de control de acuerdo con la legislación vigente.

Desarrollo alternativo de actividades económicas para disminuir la presión sobre el uso del agua (por ejemplo Agroindustria, etc):

- Sensibilización sobre el uso del agua.

- Implementación de tecnologías eficientes de riego.

4. Ordenamiento territorial: Zonificación Ecológica Económica

- Catastro de áreas de cultivo

- Zonificación de cultivos

Alternativas económicas del uso de la tierra.

Plan Director

- Difusión del Plan Director

- Menor invasión de terrenos eriazos por pequeños agricultores: (control de invasiones) (entubado agua).

5. Vulnerabilidad:

Plan de Contingencia respecto a heladas.
Sistema meteorológico de alerta temprana

- Inventario de las capacidades de comunicación existentes.

- Sistema de comunicaciones integrado.

Plan de Contingencia respecto a sequias.

Menor erosión hídrica: control de la erosión.

Análisis de peligro en Calana: defensas ribereñas. Alternativas para reducir la pérdida de agua en la quebrada de Vilavilani: (por ej. Entubado).

\section{Calidad del agua}

Estudios geológicos de contaminación

- Evaluación de la intrusión marina en La Yarada.

Diagnóstico de vertimientos de Aass

- Informe sobre puntos de vertimiento.

- Análisis de la calidad de agua.

\section{Calidad del Suelo}

Evaluación del proyecto de uso de las aguas del río Maure.

- Conseguir estudio de ATA (PET y GRT)

- Evaluación de la Contaminación (salinización) por residuos agricolas.

\section{Información y Capacitación}

Información y capacitación sobre uso sostenible de los recursos.

\section{Marco Legal}

Plan de Manejo de Cuencas aprobado por el GR Tacna:

- Aprobación por Grupo Técnico.

- Aprobación por la CAR Tacna.

- Aprobación por el GR Tacna.

Propuesta de mejora del marco legal

- Evaluación de pago por uso de agua subterránea.

- Evaluación del derecho de propiedad de terrenos en relación con la restricción de uso de agua (ATDR).

\section{Inversiones}

Plan de Cuencas en presupuesto participativo:

- Evaluación de la sostenibilidad de proyectos agrícolas.

- Mayor inversión del GR Tacna en infraestructura en las cuencas

- Asignar presupuesto para atender emergencias (fondo intangible) 


\section{Organización: Comisión Multisectorial para la gestión del Agua (ejemplo Piura).}

- Diagnóstico de la actual estructura administrativa del manejo del agua (D.S. 057 de la Ley de Aguas).

- Fortalecimiento de la Junta de Usuarios de Tacna.

Adecuada organización de usuarios

- Participación de EPS en desarrollo e inversión en la cuenca.

Sistema de gestión de cuencas:

- Mejor comunicación entre las juntas de usuarios y los órganos competentes.

\section{Marco legal sobre derechos de agua: incentivos a manejo recurso hídrico}

\section{CONCLUSIONES}

1.La intervención en las cuencas hidrográficas Maure-Uchusuma y Caplina a cargo de actores directos e indirectos lo efectúan en forma descoordinada y fragmentada.

2.A nivel de cabecera del valle de Tacna [Partidor Piedra Blanca], inadecuada distribución del agua para el uso poblacional y agrícola principalmente, con relación a las prioridades establecidas en la Ley General de Aguas.

3.Existencia de instituciones, públicas, privadas y organizaciones de usuarios no orgánica, débil autoridad y falta de representatividad multisectorial de usuarios del agua.

4. No existen Padrones de Derechos de Uso de Agua de los Distritos de Riego de la sierra legalmente constituidos, de igual manera la titularidad de sus tierras agrícolas [Acción del PROFODUA del INRENA].

5.Falta de participación de las organizaciones de usuarios en la toma de decisiones en la gestión del agua, siendo mucho menor en las zonas altas de cada sub cuencas, donde predomina la informalidad.

6. No es factible determinar la demanda potencial de recursos hidricos por parte del sector minero, en tanto no se conocen la cantidad ni calidad de las reservas de minerales y no se han constituido mesas de trabajo que permitan negociar el aporte de las empresas mineras para mejorar la oferta de los recursos hídricos del subsuelo, en el flanco occidental de la Cordillera del Barroso.
7.No se ha conformado una Comisión Técnica Especial entre Perú y Chile para caracterizar conjuntamente el acuifero La Yarada y aprovecharlo técnica y sosteniblemente.

8.Problemas de gestión en canal Uchusuma. 52 kilómetros de su longitud se desarrolla en territorio chileno, dificultando su $\mathrm{O}$ y M.

9.No se han presentado aún problemas críticos para el aprovechamiento de las aguas involucradas entre Perú y Bolivia, pero no deja de ser un problema potencial.

10.Problema entre Puno y Tacna: Puno se opone a que las aguas del sistema hidráulico Huenque Aguas Calientes, cuyos tributarios se ubican en territorio puneño, se trasvasen para dotar de agua a la Región Tacna.

11.Ordenanza Regional de Puno ha declarado que todas las aguas mencionadas son intangibles $y$, por lo tanto, quedan excluidas para ser aprovechadas por una Región distinta a la Gobierno Regional de Puno [decisión ilegal].

12.Avance de área cultivada en laderas crea conflictos por sobreutilización de RR.NN. Repercute en el comportamiento hidrológico de la cuenca.

13.La población civil organizada no está involucrada ni identificada plenamente en el uso y conservación de los recursos hidricos.

14.La producción agroindustrial en el ámbito de las cuencas hidrográficas de la Región Tacna es aún incipiente.

15.Obras construidas por el PET han deteriorado el medio ambiente de la subcuenca Uchusuma.

16. Existe sobreexplotación del acuifero La Yarada por la expansión agrícola de tierras y pozos ilegales.

17.Problema en el área de influencia de la Bocatoma de Chuschuco donde existen tomas parcelarias clandestinas, disminuyendo la oferta de agua para ciudad y valle de Tacna.

18.Las pérdidas de uso del agua de uso poblacional llega al $35 \%$.

19.Avance del proceso de desertificación y degradación en zona costera.

20. Muy deficiente operación y mantenimiento de la infraestructura hidráulica 
mayor y menor de riego [cuencas altas y valles agrícolas] respectivamente por la carencia de medios económicos.

21. Crecidas en la zona altoandina durante la estación lluviosa producen erosión lateral sobre las terrazas de pajonales y transporte de materiales finos (arcillas, arenas), gravas $y$ cantos.

22. Huaicos asociados al Fenómeno del Niño y por escorrentía concentrada en la vertiente occidental de las cuencas Caplina y Uchusuma.

23. Referente a las inundaciones: En forma general a nivel regional el período de las grandes avenidas ocurre entre los meses de enero a marzo.

24. Sequías: Su ocurrencia es especialmente frecuente y severa, por encontrarse en la cabecera del gran desierto de Atacama, constituyéndose en uno de los aspectos más críticos para el planeamiento de los recursos hídricos.

25.El efecto negativo en zona altoandina es diferente al de la costa, porque las condiciones de desarrollo agrícola son menores, pero la población es más vulnerable por su menor nivel económico y menos alternativas para reponerse de los efectos negativos de este tipo de eventos extremos.

\section{RECOMENDACIONES}

Determinar la demanda potencial de recursos hídricos por el sector minero, en función de la cantidad y calidad de las reservas de minerales determinadas en la etapa de exploración. Considerando, además, la fortaleza y oportunidad que ofrece el descubrimiento de reservas auríferas, que para su recuperación demandan limitadas cantidades de recurso hídrico.

Constituir Mesas de Trabajo Intersectoriales que permitan negociar el aporte de las empresas mineras para mejorar la oferta de los recursos hídricos del subsuelo, en el flanco occidental de la Cordillera del Barroso, y el tratamiento de las aguas subsuperficiales contaminadas de manera natural, para que alcancen calidad de uso.

\section{BIBLIOGRAFÍA}

Bosch, C. et al. (1999). Agua, saneamiento y la pobreza. World Bank.

Comision Técnica Multisectorial. Ministerios de Agricultura, Defensa, Economia y Finanzas, Energía y Minas, Vivienda, Construcción y Saneamiento, Salud y Producción. (2004). Estrategia Nacional para la Gestión de los Recursos Hídricos Continentales del Perú. Lima.

CONCYTEC. (2006). Consejo Nacional de Ciencia y Tecnologia: Programa Nacional de Ciencia y Tecnología e innovación en recursos hídricos. Lima.

Dourojeanni, Axel. (2001). El enfrentamiento de culturas y los conflictos de gestión del agua. ¿Pueden resolverse racionalmente?. Lima.

Global Water Partnership-Gwp. Comité Técnico para América del Sur. (2001). La gestión integrada de los recursos hidricos en el Perú. 96 p. Lima.

Jouravlev, Andréi. (2001). Administración del Agua en América Latina y el Caribe en el umbral del Siglo XXI. CEPAL-ECLAC. Santiago de Chile.

Masson, Luís. (2001). El recurso agua en el Perú, problemas y perspectivas. En El Reporte del Medio Ambiente en el Perú.USAID. Lima.

Peña Humberto Y Solanes Miguel. (2003). “La gobernabilidad efectiva del agua en las Américas. Un tema critico".GWP South America. CEPAL.

Toledo, Adolfo. (2007). Diagnóstico de la gestión del agua cuencas Hidrográficas Locumba Sama Caplina Región Tacna. Curso Gestión del Agua. CIP- Consejo Departamental Tacna. 


\section{ANEXO}

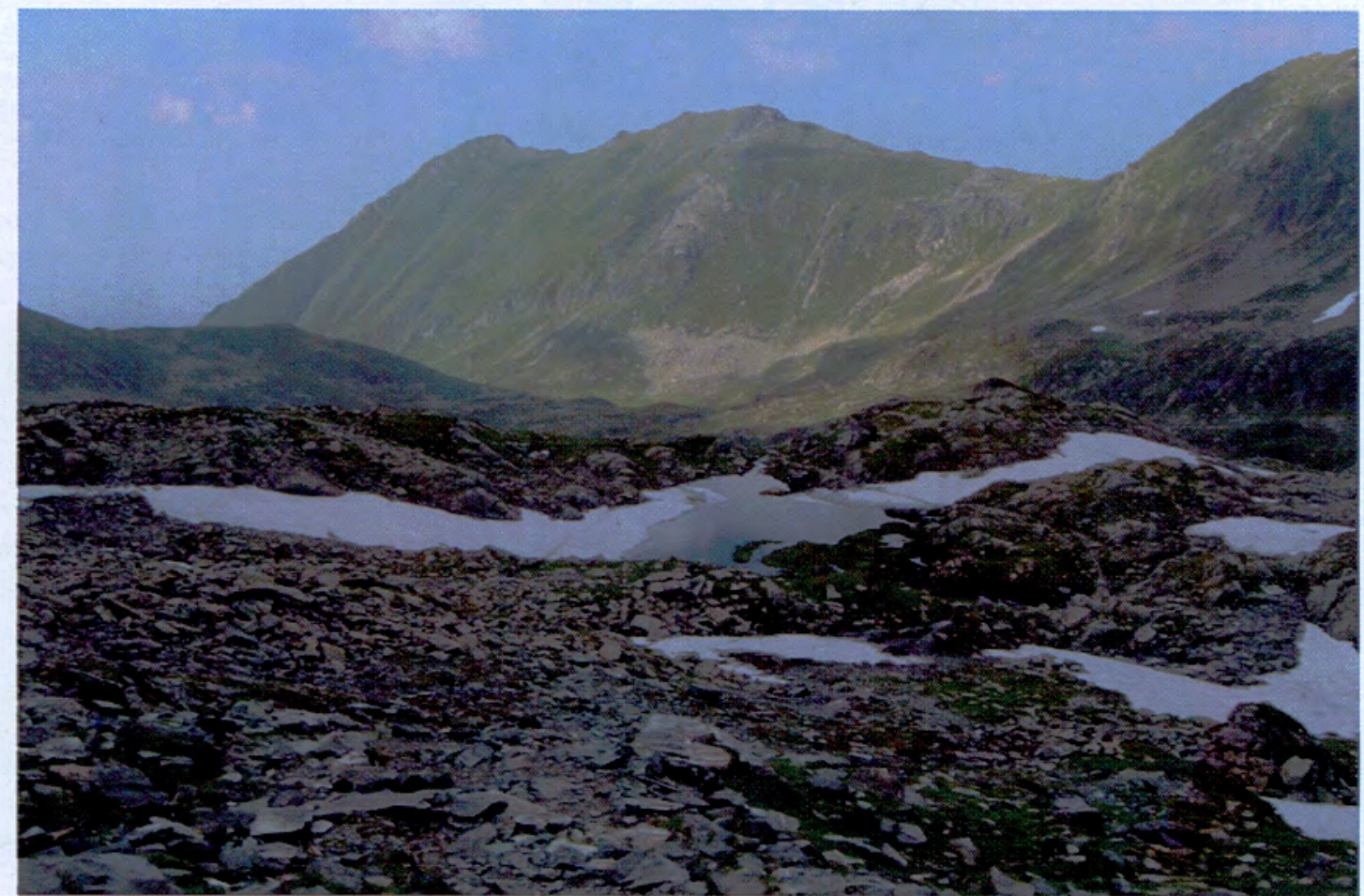

Foto 1 : Uchusuma - Caplina Región Tacna

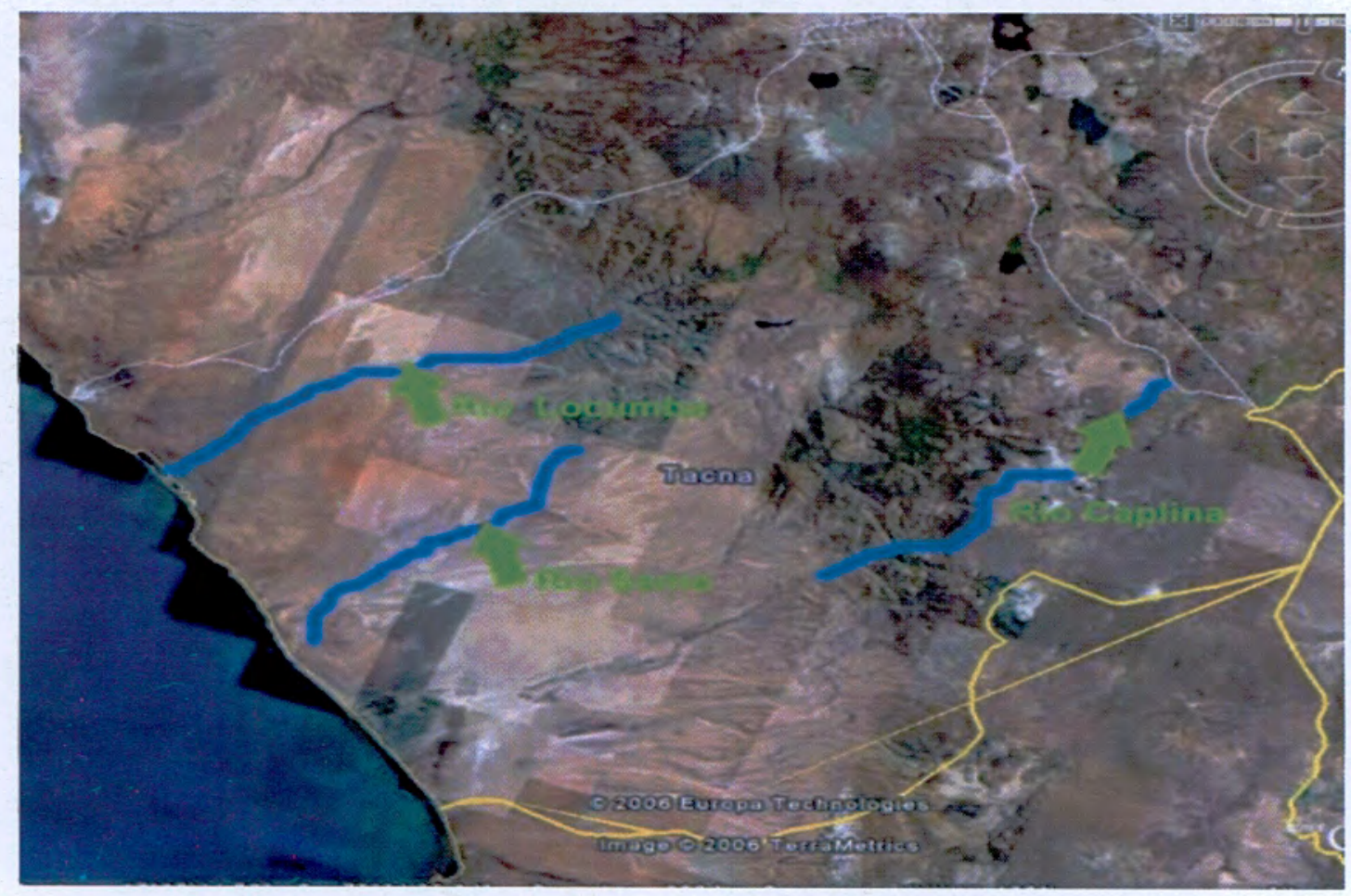

Foto 2 : Mapa cuenca maure Uchusuma - Caplina Región Tacna 